\title{
Aneurisma de arco aórtico secundario a degeneración de la media. A propósito de un caso de muerte súbita juvenil
}

\author{
Aortic arch aneurysm secondary to medial degeneration. \\ Report of a case presenting as sudden juvenile death
}

\section{Resumen}

Los aneurismas de aorta torácica son una causa significativa de muerte en los países occidentales. Frecuentemente, la muerte súbita e inesperada debida a una disección y/o rotura aguda de la aorta es la primera manifestación de una enfermedad silente que ha cursado con un periodo de dilatación aórtica progresiva.

Cuando estas situaciones se presentan en un individuo joven y sin cambios ateroscleróticos de la pared aórtica, la mayoría de las veces se sospecha la existencia de un trastorno genético. Sin embargo, existen otras posibles causas de la dilatación y posterior rotura/disección de la aorta torácica, siendo la más frecuente la degeneración de la media (DM) de origen idiopático, también denominada Síndrome de Erdheim-Gsell o Enfermedad de Erdheim.

Presentamos el caso de una muerte súbita en una mujer de 18 años, sin antecedentes médicos conocidos y sin fenotipo marfanoide, consecutiva a la rotura de un aneurisma sacular de la aorta torácica. El examen histológico confirmó la existencia de una degeneración de la capa media de la pared aórtica como único hallazgo de interés. Se realizó estudio genético de colagenopatías a la hermana de la fallecida que resultó negativo. Por todo esto, se estableció como causa de la muerte la rotura de un aneurisma de aorta torácica sobre una DM idiopática.

Palabras clave: Aneurisma de aorta torácica. Rotura. Degeneración idiopática de la capa media. Muerte súbita. Patología forense.

\section{Abstract}

Thoracic aortic aneurysms are a significant cause of death in Western countries. Frequently, sudden unexpected death due to acute dissection and/or rupture of the aorta is the first manifestation of a silent disease with progressive aortic dilatation.

These situations in young people, and without atherosclerotic changes of aortic wall, are suspicious for genetic disorders. However, there are other possible causes of the dilatation and subsequent rupture/dissection of the thoracic aorta, being the most frequent the idiopathic medial degeneration, also called Erdheim-Gsell syndrome or Erdheim Disease.

We report a case of sudden death in an 18-year-old woman with no medical history and no marfanoid habitus, secondary to the rupture of a saccular aneurysm of the thoracic aorta. Histological examination confirmed the existence of a degeneration of the media layer of the aorta, without another abnormality. Genetic studies for collagenopathies conducted in her sister were negative. The rupture of a thoracic aortic aneurysm due to idiopathic medial degeneration was established as the cause of death.

Key words: Thoracic aortic aneurysm. Rupture. Idiopathic medial degeneration. Sudden death. Forensic pathology.

\section{Introducción}

Un aneurisma es una dilatación anómala localizada en un vaso sanguíneo o en el corazón. En el caso de los aneurismas vasculares hablamos de "aneurismas verdaderos" ya que están rodeados por todas las capas de la pared arterial ${ }^{1}$. Frente a éstos, existen también los Ilamados pseudoaneurismas o "aneurismas falsos"
JV. Pachar ${ }^{1}$

JA. Hidalgo ${ }^{2}$

VA. Aguirre ${ }^{2}$

OL. Alvarado ${ }^{2}$

M. Santos ${ }^{3}$

${ }^{1}$ Patólogo forense. Departamento de Patología Forense. Instituto de Medicina Legal y Ciencias Forenses de Panamá. (República de Panamá).

${ }^{2}$ Médico residente de Medicina Forense. Instituto de Medicina Legal y Ciencias Forenses de Panamá.

(República de Panamá). ${ }^{3}$ Médico forense. Servicio de Patología Forense. IML de Sevilla (España).

Correspondencia: Dr. JV Pachar E-mail: jvpachar@gmail.com

Fecha de recepción: 14.JUN.2010 Fecha de aceptación: 16.JUN.2010 
que son dilataciones aneurismáticas que no contienen todos los elementos de dicha pared vascular, sino que su pared está compuesta primariamente por capas variables de la media o de la adventicia, trombos y estructuras adyacentes; es el caso de los aneurismas postoperatorios y de algunas formas de aneurismas traumáticos 0 de origen infeccioso ${ }^{1,2}$.

Desde un punto de vista descriptivo, los aneurismas pueden clasificarse macroscópicamente por su forma y su tamaño. Así, los aneurismas saculares son fundamentalmente esféricos, afectan sólo a un pequeño tramo de la pared vascular, su tamaño oscila entre los 5 y los $20 \mathrm{~cm}$ de diámetro y suelen estar ocupados total o parcialmente por un trombo. Por otra mes, con afectación de un segmento largo del vaso; estos aneurismas varían de diámetro (hasta $20 \mathrm{~cm}$ ) y de longitud, pueden afectar a toda la porción ascendente y transversa del cayado aórtico, así como a grandes segmentos de la aorta abdominal o incluso a las arterias ilíacas. La forma que adopte el aneurisma, sin embargo, no es en ningún caso específica de una enfermedad ni se asocia con unas manifestaciones clínicas determinadas ${ }^{1}$.

Los aneurismas de aorta torácica son unas entidades patológicas relativamente frecuentes en nuestro medio con una incidencia aproximada de $5.9 / 100.000$ personas y año, incidencia que parece estar aumentando a raíz del desarrollo de técnicas de screening más eficaces y fundamentalmente en relación al uso de técnicas de imagen mejoradas ${ }^{2,3}$. La edad media al momento del diagnóstico de la enfermedad aneurismática es de 59-69 años, aunque en los varones se diagnostica en edades más tempranas y con una predominancia de 2-4:1 frente a las mujeres. Las principales complicaciones de un aneurisma de aorta, la rotura y/o disección del

Figura 1.

Aneurisma sacular en la porción terminal del arco aórtico. Destaca un notable adelgazamiento de la pared. parte, los aneurismas también pueden ser fusifor-

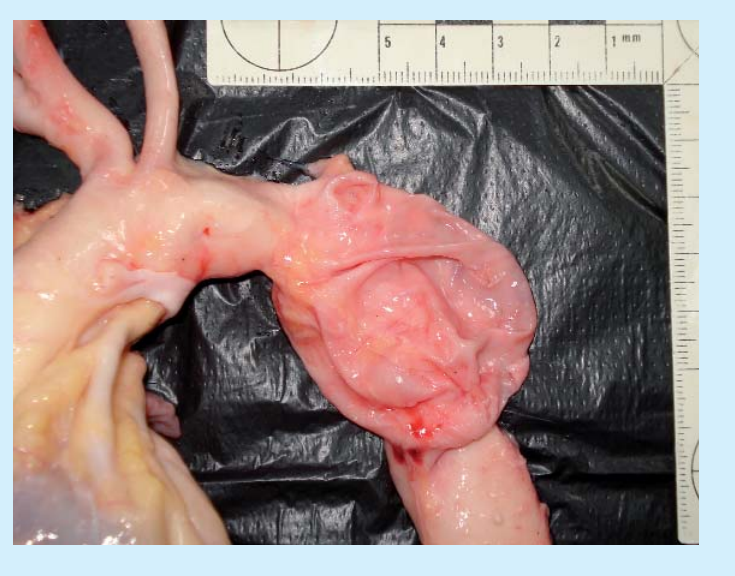

mismo, son una causa importante de morbilidad y mortalidad en los países occidentales, representando la $13^{\text {a }}$ causa de muerte en Estados Unidos ${ }^{2,4}$. Generalmente, los aneurismas de aorta torácica se localizan distalmente próximos al origen de la subclavia izquierda, aunque también pueden aparecer en el segmento ascendente del cayado donde resulta más frecuente su evolución a la disección, circunstancia que ocurre en aproximadamente el $50 \%$ de los casos. El riesgo de rotura es mayor en mujeres y en edades discretamente más tempranas de la vida (61 años) ${ }^{5}$.

Frecuentemente, la muerte súbita e inesperada debida a una disección o rotura aguda de la aorta aneurismática es la primera manifestación de una enfermedad silente que ha cursado con un periodo de dilatación aórtica progresiva ${ }^{3,6}$. Dadas las circunstancias en que se presenta el óbito, en estos casos se requiere la práctica de la autopsia médico-legal para establecer la causa y mecanismo de la muerte, pero también para hacer un diagnóstico diferencial con las entidades que subyacen bajo la formación del aneurisma y, en el caso de los cuadros hereditarios, informar a la familia y derivarla para la pertinente evaluación clínica y consejo genético ${ }^{3,6,7}$.

\section{Presentación del caso}

Mujer de 18 años con antecedentes de síncope un mes antes del evento fatal, no habiendo consultado con ningún médico. Se encontraba sentada junto a su familia en la rivera de un río y súbitamente dice sentirse mal, pierde el conocimiento y cae al agua. Es recuperada del agua inmediatamente pero fallece a los pocos minutos. Inicialmente se sospecha que pudiera tratarse de una sumersión accidental.

La autopsia se realizó con un retraso postmortem de 16 horas. Se trataba del cadáver de una mujer de hábito atlético con una talla de $1,65 \mathrm{~m}$ y $54,5 \mathrm{~kg}$ de peso aproximadamente (IMC $20 \mathrm{~kg} / \mathrm{m}^{2}$ ), sin signos externos de violencia ni hábito marfanoide.

A la apertura de la cavidad torácica se encontró un hemotórax izquierdo masivo de 3.000 cc debido a la rotura de un aneurisma sacular de la porción terminal del arco aórtico, ligeramente distal al origen de la arteria subclavia izquierda. Dicho aneurisma tenía unas medidas de 4,2 x 4,6 cm y su pared, notablemente adelgazada, a penas alcanzaba $1 \mathrm{~mm}$ de espesor (Figura 1). La línea de rotura medía $2 \mathrm{~mm}$ de longitud y se encontraba situada en la región inferoanterior del saco aneurismático.

El corazón tenía un peso de 200 g, siendo el peso esperado de 199 g, según tablas de Kitzman. A nivel 
del endocardio subaórtico se apreciaba un área clara ligeramente engrosada. Tanto las válvulas auriculoventriculares como las semilunares eran normales, al igual que el origen de las arterias coronarias.

El examen histopatológico de la pared del aneurisma mostró adelgazamiento de la pared de la aorta, a expensas de la rotura y la pérdida de las fibras elásticas y reducción del tejido muscular que se encontraba sustituido por una matriz basófila no fibrilar (Figura 2).

Ante la sospecha de la existencia de una colagenopatía atípica, es decir sin expresión fenotípica, se realizó screening genético de Síndrome de Marfan a la hermana menor de la fallecida -previo consejo genético en el Hospital del Niño-. La técnica utilizada fue la investigación genética molecular del marcador FBN1 que se amplificó por protocolo de PCR estándar, secuenciados y cuantificados mediante electroferogramas (electroforesis capilar), analizados y comparados con secuencias de referencia. El estudio resultó negativo. No se tamizaron otras enfermedades por carencia de los marcadores genéticos en ese momento.

\section{Discusión}

La historia natural de los aneurismas de aorta torácica no es bien conocida, fundamentalmente porque cursan de forma asintomática hasta que alcanzan un volumen importante que causa afectación de estructuras vecinas -compresión visceral, oclusión de ramas vasculares originando fenómenos isquémicos, irritación de raíces nerviosas o erosión de estructuras óseas próximas-, o surge una complicación como una rotura o una disección con la consiguiente hemorragia masiva, como ocurrió en el caso que presentamos $^{1,4,8}$

La elasticidad y resistencia a la tracción de la aorta dependen en su mayor parte de la capa media, formada por aproximadamente 45-50 unidades laminares de elastina, colágeno, células musculares lisas y sustancia fundamental. En la aorta ascendente el contenido en elastina es mayor, acorde con su naturaleza distensible, y va disminuyendo distalmente a lo largo de la aorta torácica descendente y la aorta abdominal ${ }^{2,4}$. La capa media también se hace más fina en los tramos distales, de modo que a nivel abdominal el número total de láminas es casi la mitad. La pared aórtica es un medio biológicamente activo, en el que las células musculares sintetizan y degradan elastina, colágeno y proteoglicanos ${ }^{2}$.

Un grado ligero de fragmentación de fibras elásticas es normal con el aumento de la edad, de hecho múltiples estudios han sugerido que el diámetro de la

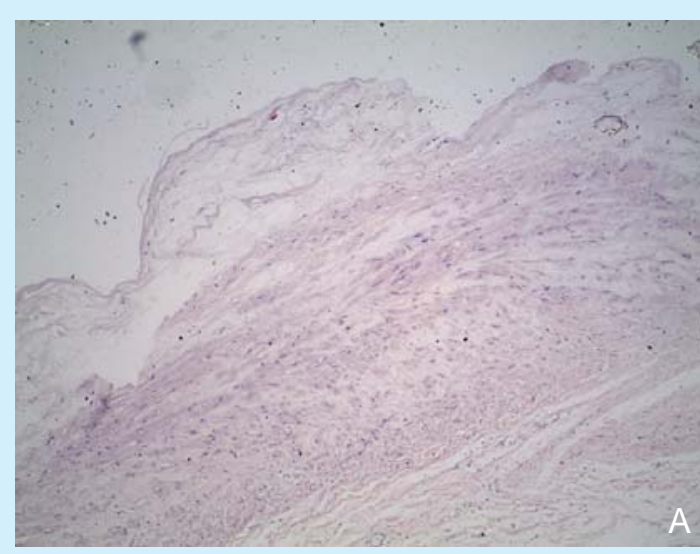

Figura 2.

Adelgazamiento de la pared de la aorta en la zona del aneurisma con rotura y pérdida de fibras elásticas $y$ reducción del tejido muscular sustituido por una matriz basófila no fibrilar. (A: HE; B: elásticas; C: tricrómico).
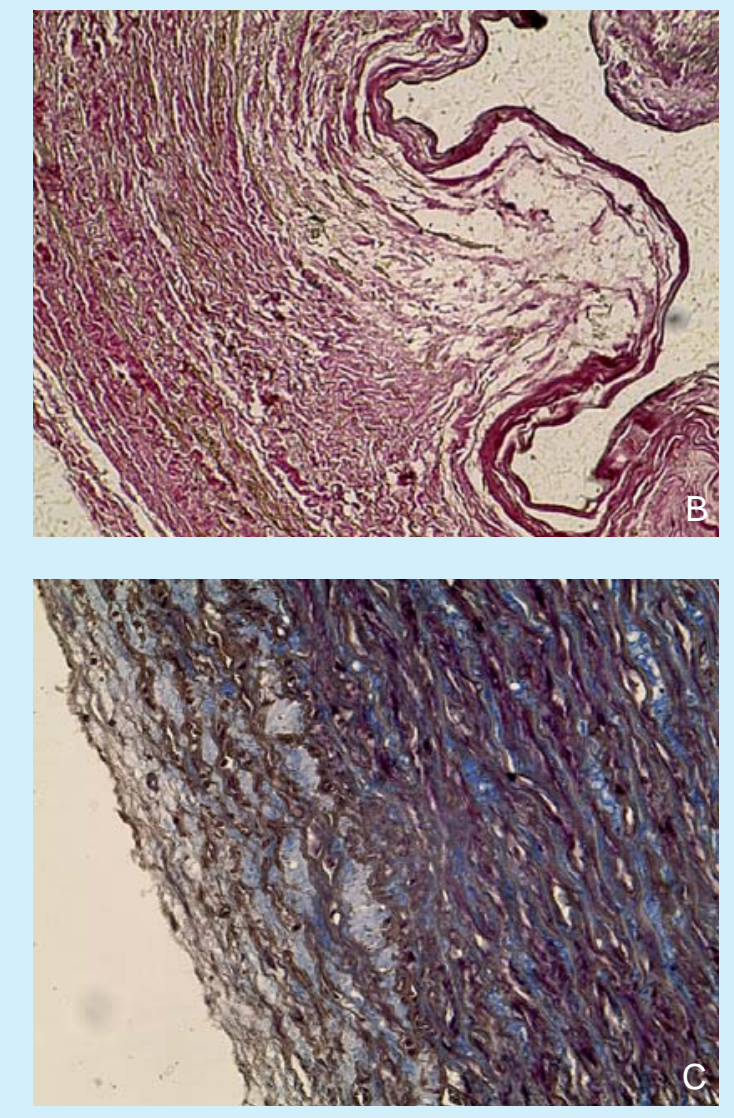

aorta ascendente y del arco aórtico crece anualmente de 0,07 a 0,2 cm. Esta dilatación, que en principio no tiene relevancia clínica, puede incrementarse y/o acelerarse hasta hacerse patológica en relación a varios factores de riesgo, como son el tabaquismo (asociado a un aumento de la concentración de enzimas elastolíticas en la pared aórtica), antecedentes familiares de aneurismas arteriales (hasta un $20 \%$ de los 
pacientes con aneurismas de aorta tiene al menos un familiar de primer grado afectado), la presencia de EPOC o la HTA ${ }^{2-4}$.

La causa más frecuente de aneurismas de la aorta torácica es la degeneración de la capa media arterial de origen idiopático, también conocida como Síndrome de Erdheim-Gsell o enfermedad de Erdheim². No obstante, la pared vascular puede resultar debilitada por otros numerosos procesos como la aterosclerosis, si bien ésta es más frecuente a nivel de la aorta abdominal -la teoría tradicional considera que el desarrollo de ateromas invasivos sobre la íntima resulta en la destrucción de fibras elásticas y células musculares lisas en la media, de modo que la pared aórtica se adelgaza, se debilita y se dilata; pero actualmente se cree que probablemente dicha aterosclerosis es un proceso concomitante que infiltra a una túnica media previamente enferma con barreras alteradas, lo que explicaría el diverso curso de la aterosclerosis a nivel de la pared aórtica: enfermedad obstructiva versus aneurismática ${ }^{1,2}$, traumatismos, enfermedades genéticas del tejido conectivo como el Síndrome de Marfan, el de Ehlers-Danlos o el de Loeys-Dietz relacionadas con defectos de las proteínas estructurales, malformaciones congénitas como la válvula aórtica bicúspide, infecciones 0 arteritis ${ }^{1,2,4,9,10}$.

Sea cual sea la causa de la debilitación y la pérdida de elasticidad de la pared aórtica, y la subsiguiente dilatación, esta situación resulta en un aumento de la tensión que soporta la pared relativa a la presión intravascular, de acuerdo con la Ley de Laplace. De modo que se amplifican los efectos perjudiciales producidos por la hipertensión sobre la pared de la aorta, llevando a un avance progresivo de la dilatación. Estos cambios patológicos pueden resultar en un acoplamiento ineficaz entre ventrículo izquierdo y aorta durante la sístole y la diástole con detrimento del flujo sanguíneo efectivo, una incompetencia de la válvula aórtica o una potencial rotura y/o disección ${ }^{2}$.

La degeneración de la media (DM), denominada frecuentemente necrosis quística de la media, es un hallazgo histopatológico presente de forma habitual en la pared de los aneurismas de aorta ${ }^{1,2,9}$. La imagen histológica típica se caracteriza por una fragmentación de las fibras elásticas y separación de los elementos elásticos y fibromusculares de la túnica media por pequeños espacios similares a hendiduras donde se pierde el tejido elástico normal. En formas avanzadas hay una pérdida dramática de elastina y células con acumulación de material basófilo amorfo que da a la media un aspecto quístico pero, aunque lo parezcan, no son verdaderos quistes. Así pues, el término necrosis quística de la media es inexacto puesto que no existen ni necrosis ni quistes. Tampoco hay inflamación ${ }^{1,2}$.

En el caso que nos ocupa, al margen de esta histología característica, no se detectaron causas subyacentes ni otras lesiones anatomo-patológicas concomitantes (no existían signos de aterosclerosis), ni un fenotipo compatible con alguna de las enfermedades del tejido conectivo referidas. Por todo esto, se concluyó que se trataba de una DM de origen idiopático. De todos modos, atendiendo a estudios recientes, se ha postulado que muchos de estos cuadros de DM idiopática pueden ser desórdenes metabólicos que aceleran la degeneración de la pared aórtica en respuesta a factores de riesgo comunes $^{2}$ o casos de Síndrome de Marfan atípico sin los rasgos fenotípicos propios del "hábito marfanoide"6; de ahí que se recomiende ser cauteloso a la hora de establecer el diagnóstico del Síndrome de Erdheim-Gsell como causa fundamental de la muerte procurando, en la medida de lo posible, completar el examen macro y microscópico con estudios genéticos que permitan descartar otras patologías silentes ${ }^{6}$.

\section{Bibliografía}

1. Schoen FJ. Vasos sanguineos. En: Kumar V, Abbas Ak, Fausto N. Robbins and Cotran. Patología estructural y funcional. $7^{\mathrm{a}}$ ed. Madrid: Ed Elsevier; 2006. p. 517-60.

2. Brinster DR, Rizzo RJ, Bolman RM. Ascending aortic aneurysms. En: Cohn LH. Cardiac surgery in the adult. $3^{a}$ ed. Nueva York: Ed McGraw Hill; 2008. p. 1223-49.

3. Ripperger T, Dieter Troger H, Schmidtke J. The genetic message of a sudden unexpected death due to thoracic aortic dissection. Forensic Sci Int 2009;187:1-5.

4. Patel HJ, Deeb GM. Ascending and arch aorta pathology, natural history, and treatment. Circulation 2008; 118:188-95

5. Donat Laporta E. Aneurismas de aorta. En: Centro de Estudios Jurídicos. Anatomía patológica macroscópica del tórax. Centro de Estudios Jurídicos. Ministerio de Justicia. Gobierno de España. Publicaciones 2004, Médicos Forenses. P. 3489-508. 
6. Klintschar M, Bilkenroth $U$, Arslan-Kirchner M, Schmidtke J, Stiller D. Marfan syndrome: clinical consequences resulting from a medicolegal autopsy of a case of sudden death due to aortic rupture. Int J Legal Med 2009;123:55-8.

7. Blanco Pampin J, Ramos Garcia O. Muerte súbita en un joven con Sindrome de Marfan. Cuad Med Forense 2005;11:317-25.

8. Pierce LC, Courtney DM. Clinical characteristics of aortic aneurysm and dissection as a cause of sud- den death in outpatients. Am J Emerg Med 2008;26:1042-6.

9. Von Kodolitsch Y, Robinson PN. Marfan syndrome: an update of genetics, medical and surgical management. Heart 2007;93:755-60.

10. Colorado Casado de Amezua A, Fernandez Bayon J. Sindrome de Loeys-Dietz. Diagnóstico diferencial en muerte súbita por disección aórtica. Rev Esp Med Legal 2009;35:70-3. 